Trinity College

Trinity College Digital Repository

Faculty Scholarship

$11-2006$

Stress Evolution in Nanocrystalline Diamond Films Produced by

Chemical Vapor Deposition

Hao Li

Brown University

Brian W. Sheldon

Brown University

Abhishek Kothari

Brown University

Zhigang Ban

University of Connecticut - Storrs

Barbara L. Walden

Trinity College, barbara.walden@trincoll.edu

Follow this and additional works at: https://digitalrepository.trincoll.edu/facpub

Part of the Physics Commons 


\title{
Stress evolution in nanocrystalline diamond films produced by chemical vapor deposition
}

\author{
Hao Li \\ Division of Engineering, Brown University, Providence, Rhode Island 02912 \\ and Department of Mechanical and Aerospace Engineering, University of Missouri-Columbia, \\ Columbia, Missouri 65203
}

Brian W. Sheldon ${ }^{\text {a) }}$ and Abhishek Kothari

Division of Engineering, Brown University, Providence, Rhode Island 02912

Zhigang Ban ${ }^{\text {b) }}$

Institute of Materials Science, University of Connecticut, Storrs, Connecticut 06269

Barbara L. Walden

Physics Department, Trinity College, Hartford, Connecticut 06106

(Received 29 October 2005; accepted 8 July 2006; published online 7 November 2006)

\begin{abstract}
Nanocrystalline diamond films were grown on silicon substrates by microwave plasma enhanced chemical vapor deposition with 1\% methane, $2 \%-10 \%$ hydrogen, and argon. High resolution transmission electron microscope images and selected area electron diffraction patterns confirm that the films consist of 10-20 nm sized diamond grains. The residual and intrinsic stresses were investigated using wafer curvature. Intrinsic stresses were always tensile, with higher $\mathrm{H}_{2}$ concentrations generally leading to higher stresses. Annealing the films in a hydrogen plasma significantly increased these stresses. These hydrogen induced changes also appear to alter stress levels and stress gradients during the growth process itself. Raman spectra revealed subtle changes in the chemical bonding that were correlated with some of the stress variations. These results suggest that grain boundary bonding and hydrogen induced reactions at the grain boundaries can influence the intrinsic stresses in nanocrystalline diamond films. (C) 2006 American Institute of Physics. [DOI: 10.1063/1.2357992]
\end{abstract}

\section{INTRODUCTION}

Gruen has shown that nanocrystalline diamond (NCD) films can be synthesized by a carbon-containing noble gas plasma. ${ }^{1}$ The high surface smoothness and extremely high hardness make these films promising candidates as hard coatings for tribological applications. ${ }^{2-5}$ NCD films also have excellent electron emission properties with potential applications for flat panel displays. ${ }^{6}$ Yang et al. have proposed NCD films as substrates for biological sensors. ${ }^{7}$ Recently, significant research has been conducted to explore potential applications in microeletromechanical system (MEMS). For this application, Espinosa et al. investigated mechanical behavior with a membrane deflection technique. ${ }^{8}$ They found that NCD films have a comparatively high fracture strength of 4.13 $\mathrm{GPa}$ and a fracture toughness of $6.8-7.3 \mathrm{MPa} \mathrm{m}^{1 / 2}$. 9,10 A fundamental understanding of residual stresses is also important for the design, fabrication, and application of NCD based devices. To obtain precise dimensional control for certain MEMS devices, it is critical to control the residual stress and the stress gradients in NCD films. In particular, stress gradients can lead to unwanted bending of the film when it is removed from the underlying substrate. ${ }^{8}$ In situations where the film is not removed from the substrate, a high residual

\footnotetext{
a) Author to whom correspondence should be addressed; electronic mail: brian_sheldon@brown.edu

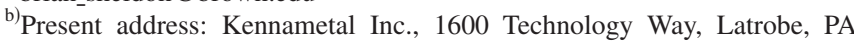
15650
}

stress can cause poor adhesion between the film and the substrate. The residual stress can also influence the long term durability of certain MEMS devices. These issues have motivated several previous investigations to reduce residual stresses. ${ }^{11-13}$

We report residual stress data for NCD films grown under a variety of different conditions. Previous work shows that NCD films can be fabricated with plasma chemical vapor deposition (CVD) using a noble gas dominant gas mixture, typically $1 \% \mathrm{CH}_{4}$ in $\mathrm{Ar}$ with or without a small amount of additional $\mathrm{H}_{2} .{ }^{1}$ This differs from the CVD of conventional microcrystalline diamond films, which are typically grown from reactant gas mixtures consisting of $\mathrm{H}_{2}$ with relatively small amounts of a carbon precursor (e.g., $0.1 \%-5 \% \mathrm{CH}_{4}$ ). Data on residual stresses in NCD are limited; however, there have been a number of studies of microcrystalline CVD diamond films. ${ }^{14-19}$ Stress generation phenomena that have been proposed in these investigations include the effects of grain boundaries, nondiamond carbon, hydrogen, and porosity. In comparison with conventional microcrystalline diamond, the defining characteristic of NCD is its fine grain size. This can be as small as $3-5 \mathrm{~nm}$ when grown without adding $\mathrm{H}_{2}$ to the gas precursors, with somewhat larger grain sizes when small amounts of $\mathrm{H}_{2}$ are added.

The residual stress in NCD films consists of intrinsic stress created during growth and thermal stress introduced by the thermal expansion coefficient difference between the film and substrate. In the present study, we systematically inves- 
TABLE I. Results for selected samples.

\begin{tabular}{|c|c|c|c|c|c|}
\hline \multirow[b]{2}{*}{ Sample } & \multicolumn{2}{|c|}{ Growth conditions } & \multirow{2}{*}{$\begin{array}{c}\text { Surface grain size*/ } \\
\text { standard deviation (nm) }\end{array}$} & \multirow{2}{*}{$\begin{array}{l}\text { Roughness, rms } \\
(\mathrm{nm})\end{array}$} & \multirow{2}{*}{$\begin{array}{l}\text { Film thickness } \\
(\mu \mathrm{m})\end{array}$} \\
\hline & $\% \mathrm{H}_{2}$ & Time (h) & & & \\
\hline D2-2h & 2 & 2 & $9.6 / 2.2$ & 28.5 & 0.6 \\
\hline D4-2h & 4 & 2 & $10.7 / 2.1$ & $\cdots$ & 1.0 \\
\hline D4-10h & 4 & 10 & $12.0 / 2.1$ & 27.1 & 4.9 \\
\hline D6-2h & 6 & 2 & $11.0 / 2.4$ & 32.9 & 1.2 \\
\hline D6-10h & 6 & 10 & $15.6 / 4.1$ & 43.1 & 7.1 \\
\hline D10-10h & 10 & 10 & $17.0 / 4.4$ & 40.8 & 7.8 \\
\hline
\end{tabular}

tigated both the average intrinsic stress and the stress gradient in NCD films. The primary aim of this research is to develop a fundamental understanding of stress evolution in NCD films.

\section{EXPERIMENT}

\section{A. Sample preparation}

The substrates were $\langle 100\rangle$ silicon wafers, 1 in. in diameter and $229 \mu \mathrm{m}$ thick, that were seeded with diamond nanopowder by (1) cleaning the silicon wafer in ultrasonic acetone bath for $5 \mathrm{~min}$, (2) putting the cleaned wafer in ultrasonic acetone bath with diamond nanopowder for about $20 \mathrm{~min}$, and (3) rinsing the treated wafer with acetone. For the deposition, the seeded substrate was put in a commercial microwave plasma reactor (AsTex HPM/M, Applied Science and Technology, Inc., Woburn, MA). All of the NCD films studied in this work were grown using the following conditions: $800 \mathrm{~W}$ microwave power, $800{ }^{\circ} \mathrm{C}$ substrate temperature, $13 \mathrm{kPa}$ pressure, $600 \mathrm{SCCM}$ (standard cubic centimeter per minute) total precursor flow rate, $1 \% \mathrm{CH}_{4}, 2 \%-10 \% \mathrm{H}_{2}$, and balance Ar. Several samples were also heat treated at $800{ }^{\circ} \mathrm{C}$ in vacuum, $\mathrm{H}_{2}$ atmosphere, or in hydrogen plasma (100 SCCM $\mathrm{H}_{2}$ and $800 \mathrm{~W}$ ) after deposition. No deposition was done without $\mathrm{H}_{2}$ because a graphite layer was deposited on the top quartz window with $\mathrm{CH}_{4}$ in a pure Ar plasma (the microwaves enter the chamber through this window).

\section{B. Microstructure characterization}

All samples were imaged with a LEO 230 scanning electron microscope (SEM), without using a conducting surface coating. The surface grain sizes of the films were estimated based on high resolution SEM images, where at least 100 grains were measured per sample. More detailed grain size information on some films was obtained by transmission electron microscopy (TEM), using a JEOL 2010. For each film, surface roughness in a $5 \times 5 \mu \mathrm{m}^{3}$, area was measured with an atomic force microscope. Raman spectroscopy was conducted with laser excitations at 632 and $488 \mathrm{~nm}$, using a laser spot size of approximately $1 \mu \mathrm{m}$.

\section{Stress measurement}

Film stress was determined by measuring the radius of curvature $R$. These data were obtained with a multibeam optical stress sensor (MOSS), where parallel laser beams are deflected by the silicon substrate and the curvature is deter- mined by monitoring the spacing between adjacent laser spots. ${ }^{20}$ Stresses were measured at room temperature and corrected for the thermal stress that occurs during postdeposition cooling $\left(-440 \mathrm{MPa}\right.$ for growth at $\left.800{ }^{\circ} \mathrm{C}\right)$.

The traditional method of converting curvature to stress is the Stoney equation. ${ }^{21}$ However, in the films studied here the film bending moment and the resultant flexure stress are not always negligible, and the film and the substrate must be treated as a composite beam. Thus with the film thickness considered, the residual stresses were calculated with the following modified version of the Stoney equation: ${ }^{14}$

$$
\langle\sigma\rangle=\frac{M_{s} H^{2}}{6 R h}\left(1+4 \frac{M_{f} h}{M_{s} H}+\frac{h}{H}\right),
$$

where $\langle\sigma\rangle$ is the average in-plane stress over the thickness of the film and $M_{s}, H, M_{f}$, and $h$ are the biaxial moduli and thickness of the substrate and the film, respectively. It is convenient to report data in terms of the stress thickness,

$$
\langle\sigma\rangle h=\int_{0}^{h} \sigma(z) d z
$$

where $\sigma(z)$ is the average in-plane stress at a given distance $z$ from the film/substrate interface. The film thicknesses were obtained from SEM images of cross-sectional specimens.

The stress values were calculated using a Young's modulus of $970 \mathrm{GPa}$ and a Poisson ratio of 0.07 , which are reported values for ultrananocrystalline diamond (UNCD) films grown at Argonne National Laboratory. ${ }^{8-10}$ A similar behavior is expected for our films. A different biaxial elastic modulus will alter the calculated value, but this difference does not influence any of the general conclusions obtained from these data. For example, a 4\% decrease in Young's modulus generates only $0.2 \%$ and $2 \%$ change, respectively, in the calculated stress values for D2-2h and D6-14h in Table I (the thinnest and thickest films studied here). Another possible source of error in applying Eq. (1) is the inelastic deformation of the $\mathrm{Si}$ substrate. Previous experiments and analysis indicate that this effect will be relatively small for most of the films studied here. ${ }^{22}$

The average stress gradient was estimated by measuring the curvature of films released from the substrate. The silicon substrate was removed by etching with $13 \mathrm{M} \mathrm{KOH}$ at $80^{\circ} \mathrm{C}$. The curvature of the remaining film was then measured close to the middle of $10 \mathrm{~mm}$ long pieces, to minimize edge ef- 

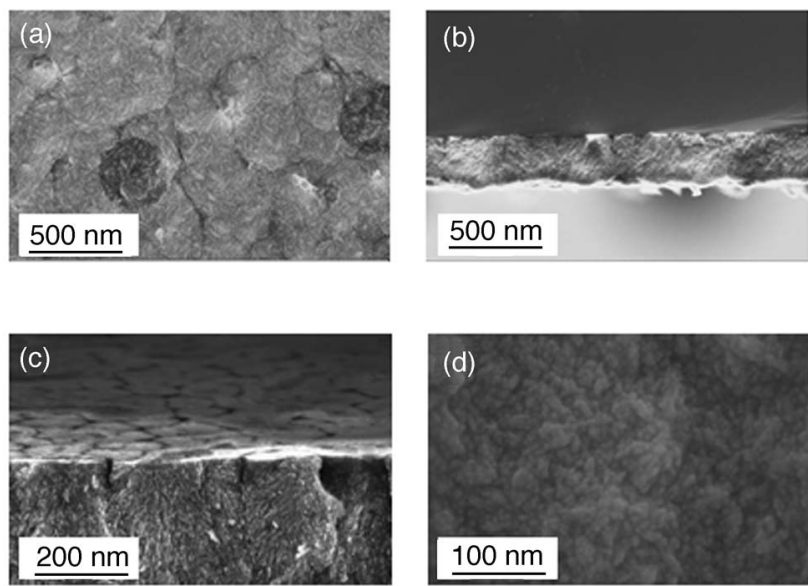

FIG. 1. Scanning electron micrographs of nanocrystalline diamond films [(a) and (b)] D2-2h (2\% H2 for $2 \mathrm{~h})$ and [(c) and (d)] D4-10h (4\% H2 for $10 \mathrm{~h}$ ). Images (a) and (d) show surface morphology. Images (b) and (c) show cross-sectional morphology.

fects. The curvature (inverse of radius $R_{f}$ ) $\kappa$ of a free standing film has the following relationship with the processinduced strains $\varepsilon(z):^{23}$

$$
\kappa=\frac{-6}{h^{2}} \int_{0}^{h} \varepsilon(z) d z+\frac{12}{h^{3}} \int_{0}^{h} z \varepsilon(z) d z .
$$

And the strain in the film is related to the stress through linear elasticity, $\sigma(z)=(E /(1-v)) \varepsilon(z)$, where $E$ is Young's modulus and $\nu$ is Poisson's ratio. Curvature based measurements with Eqs. (1)-(3) were the primary means used to study stress evolution during the deposition process.

\section{RESULTS}

\section{A. Basic characterization}

Table I presents a summary of the growth conditions, grain size, roughness, and the film thickness of selected samples. Note that very small grains might not be observable by SEM (resolution: $<3 \mathrm{~nm}$ ), such that the reported grain sizes are probably overestimated. At least 100 grains were sized for each sample. As a general trend, films with a larger grain size tend to have a rougher surface. All of the conditions in Table I produced nanocrystalline diamond. This is consistent with previous microwave plasma enhanced chemical vapor deposition (MPCVD) films. ${ }^{24}$ In hot filament CVD, the transition from nanocrystalline to microcrystalline diamond occurs at lower $\mathrm{H}_{2}$ levels $[\sim 10 \%$ (Ref. 25 ) and $\sim 4 \%$ (Ref. 26)].

Table I shows that the grain size increases with higher $\mathrm{H}_{2}$ concentration, roughly doubling when $\mathrm{H}_{2}$ increases from $2 \%$ to $10 \%$. The grain size also increases with film thickness (i.e., at longer deposition times), a behavior which is observed in many polycrystalline films. However, the correlation between $\mathrm{H}_{2}$ and grain size is a dominant effect that has been carefully documented by others. ${ }^{24-26}$

The films deposited for a short time exhibited a columnar structure, where each column contains thousands of nanosized subgrains. For example, Figs. 1(a) and 1(b) show SEM images of the sample deposited with $2 \% \mathrm{H}_{2}$ for $2 \mathrm{~h}$.
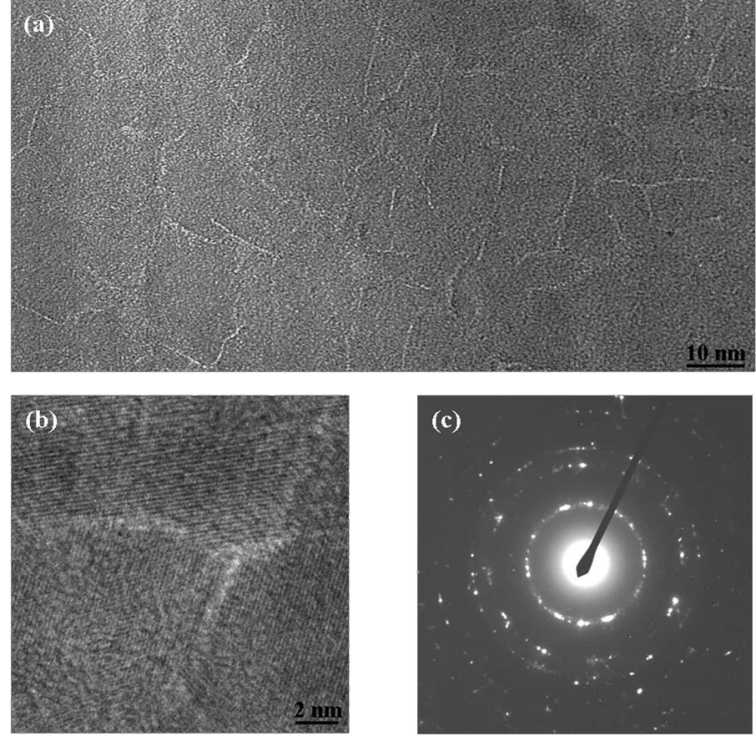

FIG. 2. Transmission electron micrographs $[(a)$ and (b) $]$ and electron diffraction pattern (c) of nanocrystalline diamond film fabricated with $10 \% \mathrm{H} 2$ for $10 \mathrm{~h}$. (D10-10h).

Here the columns are roughly $1 \mu \mathrm{m}$ in diameter. Both plan view and cross-sectional SEM images show dark circular regions which are similar in size to the diamond columns. For the diamond films grown for $2 \mathrm{~h}$ and higher $\mathrm{H}_{2}$ concentrations, the plan view SEM images looks very similar to Fig. 1(a) except that no dark regions were found. Figure 1(c) shows the back of a film delaminated from silicon substrate. In this image the columnar structure and column boundaries are clearly evident close to the silicon/diamond interface, whereas this columnar structure disappears in material that is several microns away from the diamond/silicon interface. Figure 1(d) shows an SEM image that was used to measure the surface grain size.

The $\mathrm{H}_{2}$ effect on deposition rate was also investigated. At higher $\mathrm{H}_{2}$ concentration the deposition rate increased over the $2 \%-10 \% \mathrm{H}_{2}$ range that was studied here. Using a similar CVD system, Zhou et al. observed that this trend continues with higher $\mathrm{H}_{2}$ concentration until a maximum deposition rate was reached at $1.2 \mu \mathrm{m} / \mathrm{h}$ with $40 \% \mathrm{H}_{2}$ in the $\mathrm{CH}_{4} / \mathrm{Ar}$ plasma. ${ }^{24}$ Jones et al. observed a maximum deposition rate of $1.9 \mu \mathrm{m} / \mathrm{h}$ with $50 \% \mathrm{H}_{2}$ in a hot filament reactor. $^{25}$

Figure 2 shows the plan view TEM images, including high resolution transmission electron microscopy (HRTEM) and selected area electron diffraction pattern of the diamond film deposited with $10 \% \mathrm{H}_{2}$ for $10 \mathrm{~h}$. The diamond $\{111\}$ lattice planes (lattice spacing $d_{111}=0.206 \mathrm{~nm}$ ) could be observed in HRTEM. The grain boundary thickness was $0.3-0.5 \mathrm{~nm}$. The selected area electron diffraction (SAED) pattern in Fig. 2(c) matches with diamond, while the diffraction rings corresponding to the graphite lattice were not observed. Previous work shows that this type of SAED pattern is indicative of films that are predominantly diamond. ${ }^{1}$ In particular, the TEM characterization of the NCD films presented in this study are very similar to UNCD fabricated at 


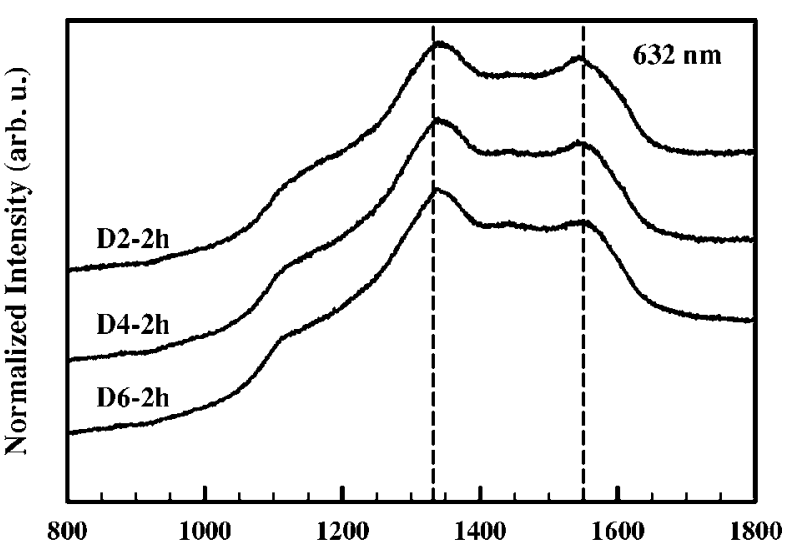

(a)

Raman Shift $\left(\mathrm{cm}^{-1}\right)$

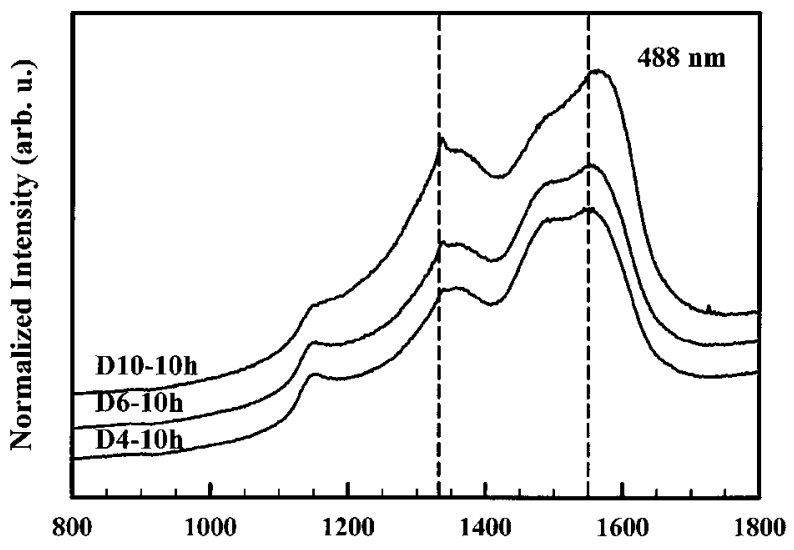

(b)

Raman Shift $\left(\mathrm{cm}^{-1}\right)$

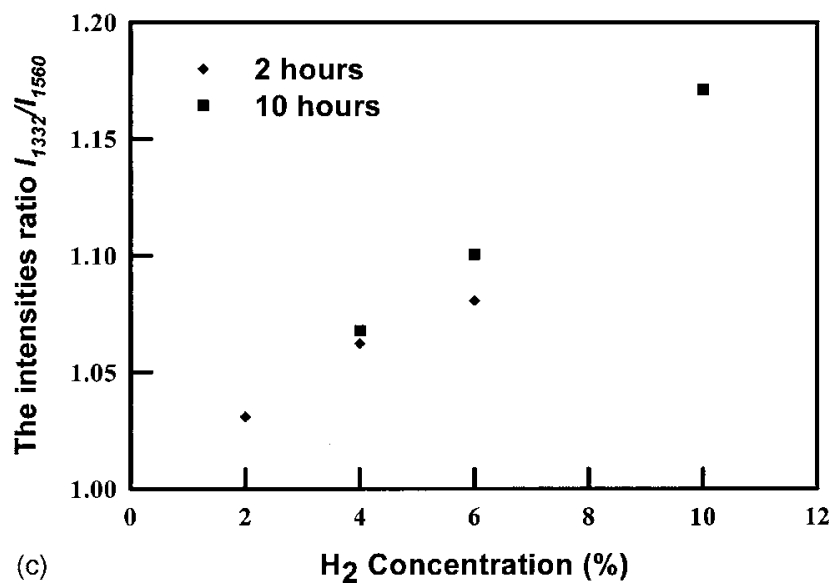

FIG. 3. Raman spectra of nanocrystalline diamond films. (a) $632 \mathrm{~nm}$ Raman spectra, (b) $488 \mathrm{~nm}$ Raman spectra, and (c) intensity ratio $I_{1332} / I_{1560}$ (based on $632 \mathrm{~nm}$ Raman spectra) vs $\mathrm{H}_{2}$ concentration.

Argonne National Laboratory except that our NCD films have somewhat larger grain sizes due to the extra $\mathrm{H}_{2}$ in the plasma. ${ }^{1,27,28}$

Raman spectroscopy is frequently used to characterize CVD diamond films, largely because it can distinguish between the different allotropes of carbon. Raman scattering in the visible range is 50-230 times more sensitive to the presence of $s p^{2}$-bonded carbon than $s p^{3}$-bonded carbon which makes it a very useful probe of the nondiamond phase in diamond thin films. ${ }^{29,30}$ Figure 3(a) shows Raman results us- ing a $632 \mathrm{~nm}$ laser excitation wavelength, for NCD films grown for $2 \mathrm{~h}$ with $2 \%, 4 \%$, and $6 \% \mathrm{H}_{2}$. These spectra show two broad peaks centered at $\sim 1340$ and at $\sim 1550 \mathrm{~cm}^{-1}$, the $D$ and $G$ peaks characteristic of disordered $s p^{2}$-bonded carbon. Recent work on NCD by Birrell et al. have employed both visible and UV Raman. ${ }^{31}$ In visible Raman spectra collected at $632 \mathrm{~nm}$, they attributed these bands to $s p^{2}$-bonded carbon at grain boundaries, rather than to graphite in the bulk structure. This is also consistent with other work on NCD. ${ }^{1,27,28,32,33}$

Figure 3(b) shows $488 \mathrm{~nm}$ Raman results for NCD films grown for $10 \mathrm{~h}$ with $4 \%, 6 \%$, and $10 \% \mathrm{H}_{2}$. At this higher laser excitation frequency the Raman cross section for scattering from $s p^{2}$-bonded carbon is decreased, compared to the spectra shown in Fig. 3(a). In Fig. 3(b) the sharper diamond $\left(1332 \mathrm{~cm}^{-1}\right)$ peak can be distinguished from the broader graphitic $D$ band $\left(\sim 1350 \mathrm{~cm}^{-1}\right)$. Birrell et al. suggested that the presence of a $1332 \mathrm{~cm}^{-1}$ peak in the Raman spectrum of similar films is associated with small quantities of microcrystalline diamond and that the fraction of microcrystalline diamond in the film increases with the $\mathrm{H}_{2}$ content of the plasma. ${ }^{31}$ Well-defined peaks at 1140 and $1480 \mathrm{~cm}^{-1}$ are also evident in the $488 \mathrm{~nm}$ spectra; these peaks are also visible as weak shoulders in the spectra taken at $632 \mathrm{~nm}$. These two features, often observed in NCD films, have been attributed to trans- $(\mathrm{CH})_{X} \quad$ (trans-polyacetylene) at grain boundaries. $^{32,34,35}$ Figure 3(b) shows that the 1140 and $1480 \mathrm{~cm}^{-1}$ peaks decrease in intensity as the hydrogen content of the plasma increases. This trend is consistent with assigning these peaks to material at grain boundaries, since additional hydrogen in the plasma increases the grain size (hence decreasing the amount of grain boundary material).

The intensity ratio $I_{1332} / I_{1560}$ of the $D$ and $G$ bands is sometimes used to characterize $s p^{2}$ carbon in diamond. ${ }^{36-38}$ However, the intensities of these two peaks were difficult to assess here because of the high degree of overlap between Raman bands in the $1200-1600 \mathrm{~cm}^{-1}$ region of the spectrum. The ratio was estimated by performing a linear fit to remove the sloping background (due to photoluminescence) from each spectrum, then getting the ratio of the resulting Raman intensities at the peaks corresponding to the positions of the $D$ and $G$ bands. No peak fitting was performed. The results for the $632 \mathrm{~nm}$ spectra in Fig. 3(c) indicate that the $I_{1332} / I_{1560}$ ratio increases as the hydrogen content in the plasma increases. These data are plotted for a series of films deposited at different times, at hydrogen concentrations between $2 \%$ and $10 \%$. In most cases multiple spectra were recorded for each film.

\section{B. Intrinsic stress}

As noted in Sec. II, Eq. (1) was used to determine the average film stress $\langle\sigma\rangle$ as a function of time. Figure 4(a) shows that the intrinsic stress generally increases with both $\mathrm{H}_{2}$ concentration and deposition time. Also, the stress approaches a steady-state value at sufficiently long deposition times. A similar effect was also observed for conventional diamond films grown in the same MPCVD system with $1 \%$ $\mathrm{CH}_{4}$ in $\mathrm{H}_{2}$, where grain sizes and intrinsic stresses are 

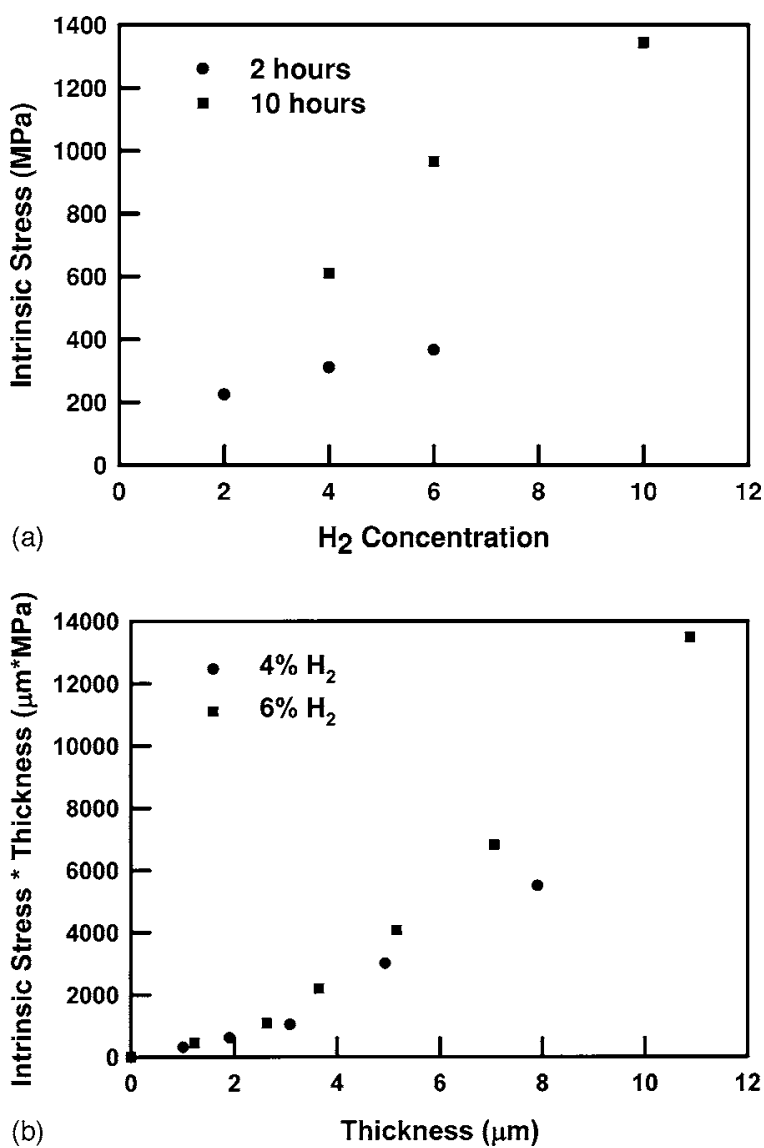

FIG. 4. (a) Intrinsic stress of nanocrystalline diamond films vs $\mathrm{H}_{2}$ concentration. (b) Intrinsic stress thickness vs thickness plot.

larger. ${ }^{15}$ In these microcrystalline diamond films, the intrinsic stress appears to be unaltered by mechanisms that occur after deposition (e.g., grain growth, grain boundary diffusion, phase transformations, etc). ${ }^{15,16}$ This makes it possible to obtain the stress profile $\sigma(z)$ as the slope of the stress thickness $(\langle\sigma\rangle h)$ versus $h$ plot (see Ref. 39 and Sec. IV B below). An example of this type of plot for the NCD films grown at $4 \%$ and $6 \% \mathrm{H}_{2}$ is shown in Fig. 4(b).

If the growth stress for each incremental layer of material is locked in as it is deposited and if the elastic properties of the film do not vary with thickness, then the slope data from Fig. 4(b) can be inserted into Eqs. (3) and (4) to predict the curvature of the corresponding free standing film. Using this approach with the data in Fig. 4(b) predicts curvatures that are significantly different from measured values that were obtained by dissolving the Si substrate. For example, with the film deposited for $10 \mathrm{~h}$ at $4 \% \mathrm{H}_{2}$, the predicted value of $\kappa^{-1}=4 \mathrm{~mm}$ does not agree with the measured value of $\kappa^{-1}=29 \mathrm{~mm}$. This large discrepancy suggests that the NCD films undergo additional stress-inducing change after material is initially deposited. In other words, as new growth occurs on the top surface, it appears that the underlying film material is altered in a way that reduces the stress variation across the film. This conclusion is discussed in more detail below (see Sec. IV B).

Annealing studies were conducted to further demonstrate that the stress in the underlying film varies after the material is first deposited. Heating NCD films in vacuum or $\mathrm{H}_{2}$ atmo-

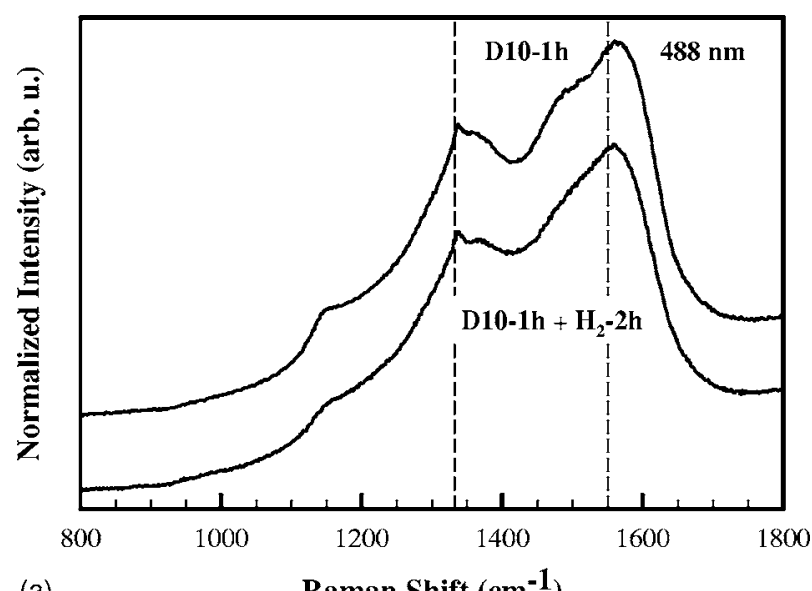

(a)

Raman Shift $\left(\mathrm{cm}^{-1}\right)$

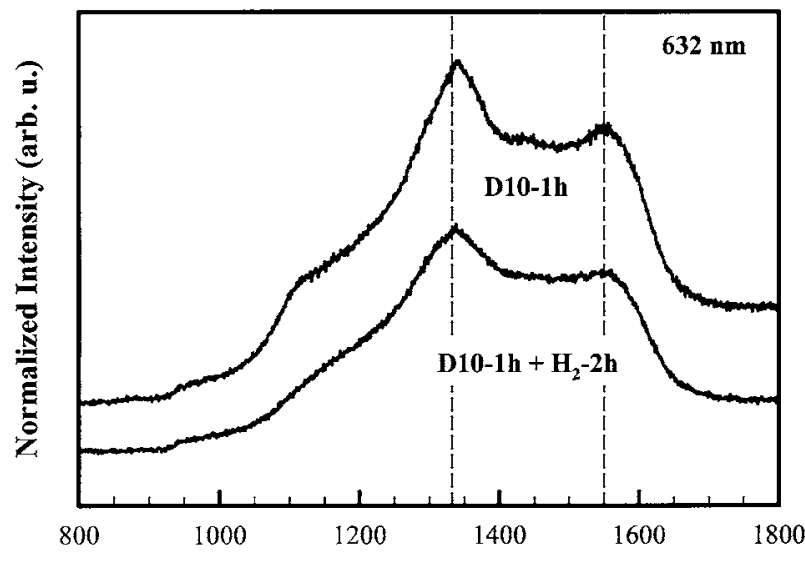

(b)

Raman Shift $\left(\mathrm{cm}^{-1}\right)$

FIG. 5. Raman data for films grown with $10 \% \mathrm{H}_{2}$ for $1 \mathrm{~h}$, before and after annealing in a hydrogen plasma at $800{ }^{\circ} \mathrm{C}$ : (a) $488 \mathrm{~nm}$ spectra, and (b) $632 \mathrm{~nm}$ spectra.

spheres at $800{ }^{\circ} \mathrm{C}$ for $2-4 \mathrm{~h}$ did not produce a discernible change in curvature (i.e., stress). However, pure hydrogen plasma annealing right after the deposition produced significant increases in the stress. For example, a NCD film deposited at $6 \% \mathrm{H}_{2}$ for $2 \mathrm{~h}$ showed an intrinsic stress change from 367 to $451 \mathrm{MPa}$ after it was exposed to a half hour postdeposition hydrogen plasma annealing. A NCD film deposited at $10 \% \mathrm{H}_{2}$ for $1 \mathrm{~h}$ showed an intrinsic stress change from 403 to $638 \mathrm{MPa}$ after a $2 \mathrm{~h}$ postdeposition hydrogen plasma annealing. These experiments indicate that atomic hydrogen affects the NCD film stress while molecular hydrogen does not. During the plasma annealing, our experiments indicate that the amount of etching that occurs is very small, within the accuracy of our film thickness measurements $( \pm 0.2 \mu \mathrm{m})$. Thus, changes in the film thickness have a negligible impact on our interpretation of the measured stressthickness values. Also, if direct etching did decrease the film thickness, this would reduce the stress thickness, in contrast to the increasing stress thickness that was observed in our experiments.

Raman spectra collected before and after the plasma annealing are shown in Fig. 5. The $488 \mathrm{~nm}$ spectra in Fig. 5(a) suggests that plasma annealing may decrease the relative intensity of the $1340 \mathrm{~cm}^{-1}$ band, relative to the diamond $1332 \mathrm{~cm}^{-1}$ band. Both the 488 and $632 \mathrm{~nm}$ spectra show that 
annealing decreases the trans-polyacetylene bands at 1140 and $1480 \mathrm{~cm}^{-1}$. The Raman results and the stress increases during the plasma annealing provide a possible explanation for the stress analysis of free standing films (discussed further below).

\section{DISCUSSION}

Three principal results reported in Sec. III are particularly relevant to understanding and controlling intrinsic stresses in NCD: (1) the intrinsic stress is tensile and increases with the hydrogen content of the plasma, (2) a comparison between MOSS data and the curvature of free standing films demonstrates that the film stress varies after material is deposited, and (3) hydrogen plasma annealing produces a significant increase in the tensile stress (whereas the film stresses are not noticeably affected by heat treatments in either vacuum or $\mathrm{H}_{2}$ ). Viewed together, these results indicate that hydrogen-related chemistry plays an important role in tensile stress generation. In the sections below the plasma annealing results are considered first, because this phenomenon also appears to affect stress evolution during growth, as discussed in the subsequent sections.

\section{A. Plasma annealing}

The tensile stresses observed during plasma annealing imply that reactive hydrogen causes densification of the film material. One possibility is that plasma annealing converts $s p^{2}$ carbon to $s p^{3}$ carbon. This has been observed in diamondlike carbon (DLC), where Raman spectra indicate that an atomic hydrogen exposure produced an increase in $s p^{3}$ character $\left(1332 \mathrm{~cm}^{-1}\right)$ that was not observed with vacuum anneals. ${ }^{40}$ From the Raman results in Fig. 5, it is not clear whether annealing produces any discernible change in the $s p^{2}$ content (i.e., the $\sim 1340$ and $\sim 1550 \mathrm{~cm}^{-1}$ bands ${ }^{30}$ ). However, this does not preclude the possibility of $s p^{2}$ to $s p^{3}$ conversion, since these changes may be relatively subtle. The results in Fig. 5 do show that plasma annealing decreases the trans $-(\mathrm{CH})_{X}$ bands $\left(1140\right.$ and $\left.1480 \mathrm{~cm}^{-1}\right)$. A similar behavior has been observed by others in nanocrystalline diamond films after $\mathrm{H}_{2}$ plasma annealings. ${ }^{41}$ All of these results suggest that more detailed studies of the hydrogen induced reactions are warranted.

Grain boundaries are a likely location for the two mechanisms described above [i.e., the $s p^{2}$ to $s p^{3}$ conversion and the removal of trans- $\left.(\mathrm{CH})_{X}\right]$. In NCD films, existing evidence based primarily on electron energy loss spectra suggests that $s p^{2}$ carbon is preferentially located at grain boundaries. ${ }^{42}$ The 1140 and $1480 \mathrm{~cm}^{-1}$ bands seen in the Raman spectra have also been associated with grain boundaries. ${ }^{34}$ Because of the very small grain sizes in NCD, changes in the grain boundary chemistry should be able to induce significant stresses. To demonstrate that this is feasible, consider the following rough estimate based on the conversion of $s p^{2}$ to $s p^{3}$ carbon. A recent tight-binding pseudopotential calculation ${ }^{43}$ predicts that NCD grown in $\mathrm{CH}_{4} / \mathrm{Ar}$ chemistry will have $\sim 0.356 \mathrm{~nm}$ wide grain boundaries with $\sim 40 \%-80 \% s p^{2}$ bonding. To roughly approximate the proposed densification due to a hydrogen induced $s p^{2}$ to $s p^{3}$ conversion, we consider a simplified film structure where $L$ and $b$ are the grain and grain boundary dimensions, respectively. The observed change in strain can be approximated as $\Delta \varepsilon=\Delta b / L$, where $\Delta b$ is the change in the grain boundary width (i.e., this assumes that changes in the undeformed lattice only occur in the grain boundaries). With this approach, the measured stress change of $235 \mathrm{MPa}(\Delta \varepsilon=2.2$ $\times 10^{-4}$ ) for one sample after $2 \mathrm{~h}$ plasma annealing corresponds to $\Delta b=0.0033 \mathrm{~nm}$. This is less than a $1 \%$ change in $b$, based on the grain boundary width of $\sim 0.356 \mathrm{~nm}$ that is cited above. A chemically induced strain of this magnitude is clearly plausible. For comparison, the atomic volume change going from bulk graphite to diamond is $36 \%$ (i.e., a linear contraction of 14\%). This comparison suggests that a $235 \mathrm{MPa}$ stress increase could be produced with an $s p^{2}$ to $s p^{3}$ conversion occurring in less than 5\% of the grain boundary atoms. While this simple estimate is unlikely to be quantitatively accurate, it demonstrates that the proposed mechanism is plausible. A more precise analysis of the volume contraction due to changes in grain boundary structure requires more detailed information about $s p^{2}$ bonding, the removal of trans- $(\mathrm{CH})_{X}$, and the possible effects of hydrogen incorporation.

We have also investigated similar heat treatments with traditional diamond films fabricated by MPCVD with $1 \%$ $\mathrm{CH}_{4} / \mathrm{H}_{2}$ chemistry. ${ }^{16,17}$ These films do not exhibit discernible stress changes during annealing in $\mathrm{H}_{2}$ or hydrogen plasma atmospheres. However, if stress variations in NCD and microcrystalline diamond were to occur by the same grain boundary mechanism, the effect would be negligible in microcrystalline diamond because of the large decrease in the number of grain boundary atoms in larger grained materials. For example, the order of magnitude approximation used above to obtain $\Delta b$ for NCD suggests hydrogen induced stress changes of less than $4 \mathrm{MPa}$ when the grain size increases to $1 \mu \mathrm{m}$. It is also possible that NCD and microcrystalline diamond have grain boundaries with significantly different crystallographic orientations and chemistry.

The apparent relationship between atomic hydrogen and tensile stress during plasma annealing suggests that the same mechanism could have a significant impact on stress evolution during NCD film growth. The principal observed similarity between annealing in the hydrogen plasma and growth with higher $\mathrm{H}_{2}$ levels is that both processes lead to higher tensile stresses. Raman peak intensities are also potentially consistent with lower $s p^{2}$ content in films grown with more $\mathrm{H}_{2}$, although this assessment is uncertain [see the discussion of Fig. 3(c) in Sec. III]. In addition to these similarities, the changes in the film caused by plasma annealing provide a possible explanation for the observation that the stress in the deposited material changes during subsequent deposition. This is considered in more detail below.

\section{B. Stress gradients}

To analyze the discrepancy between the free standing film curvature and the slope of the stress-thickness data, consider a general description of the stress profile during film growth, 


$$
\sigma(z, t)=\sigma_{o}(z)+\int_{t_{o}=z / u}^{t} \sigma_{t}(z, \hat{t}) d \hat{t},
$$

where

$$
\sigma_{t}(z, t)=\left(\frac{\partial \sigma}{\partial t}\right)_{z},
$$

where $t_{o}(z)$ is the time where the growth surface first reaches the position $z$ [i.e., $z=h\left(t_{o}\right)=u t_{o}$ for constant $u$ ], and $\sigma_{o}(z)$ is the initial growth stress produced as material is deposited on the film surface [i.e., at the point where $z=h\left(t_{o}\right)$, such that $\left.\sigma_{o}(z) \equiv \sigma\left(h\left(t_{o}\right), t_{o}\right)\right]$. When $z$ exceeds the film thickness, $z$ $>h, \sigma$ is not defined (i.e., at $t<t_{o}$ ). For deposition after $t_{o}$, material at a particular position $z$ will be underneath the growth surface [i.e., $z<h(t)]$. Thus the integral in Eq. (4) describes postdeposition changes in stress occurring at position $z$ [i.e., at $t>t_{o}(z)$, while additional diamond is being deposited above this material].

As noted in Sec. II C, curvatures are often reported in terms of the stress thickness. With this in mind, the MOSS data at time $t$ can be considered by inserting Eq. (4) into Eq. (2) to obtain

$$
\begin{aligned}
& \langle\sigma\rangle h=\int_{0}^{h(t)} \sigma_{o} d \hat{z}+\int_{0}^{h(t)}\left[\int_{t_{o}=\hat{z} / u}^{t} \sigma_{t}(\hat{z}, \hat{t}) d \hat{t}\right] d \hat{z}, \\
& \frac{d(\langle\sigma\rangle h)}{d t}=u \sigma_{o}(h(t))+\int_{0}^{h(t)} \sigma_{t}(\hat{z}, t) d \hat{z} .
\end{aligned}
$$

Combining Eqs. (4) and (6) then gives the following description of the stress profile:

$$
\sigma(z, t)=\sigma_{\text {slope }}(z)-\frac{1}{u} \int_{0}^{z} \sigma_{t}\left(\hat{z}, t_{o}(z)\right) d \hat{z}+\int_{t_{o}(z)}^{t} \sigma_{t}(z, \hat{t}) d \hat{t},
$$

where

$$
\sigma_{\text {slope }}(z)=\frac{1}{u}\left[\frac{d(\langle\sigma\rangle h)}{d t}\right]_{t=t_{o}(z)} .
$$

The first integral in Eq. (7) describes changes in the underlying film, at the time $\left(t_{o}\right)$ where material at height $z$ is being deposited. The second integral describes the stress change in the material at height $z$ during subsequent deposition [i.e., at $\left.t>t_{o}(z)\right]$. MOSS data provides a direct measure of $\sigma_{\text {slope }}$, since this quantity is the slope of the stress thickness versus thickness data. If there is no postdeposition change in the stress, then $\sigma_{t}=0$ for all $z$ and $t$, and Eqs. (6) and (7) show that $\sigma(z, t)=\sigma_{o}(z)=\sigma_{\text {slope }}(z)$. The data in Sec. III B indicates that this assumption is not valid in NCD films [i.e., inserting measured $\sigma_{\text {slope }}(z)$ data into Eq. (3) predicts $\kappa$ values that are significantly larger than $\kappa$ values obtained from direct measurements]. This discrepancy implies that the stress in the underlying film varies when additional material is added during subsequent deposition (i.e., $\sigma_{t}$ is not zero).

The observed increase in tensile stress due to plasma annealing provides a plausible explanation for the nonzero values of $\sigma_{t}$. The apparent hydrogen induced phenomena

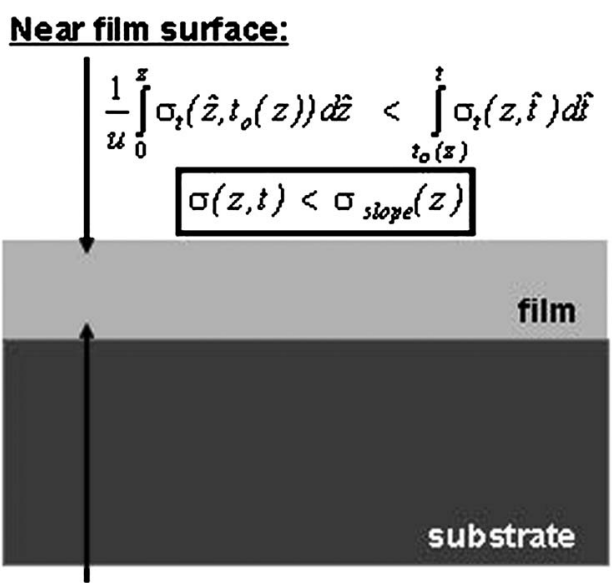

Near interface with substrate:

$$
\begin{gathered}
\frac{1}{u} \int_{0}^{z} \sigma_{z}\left(\hat{z}, t_{0}(z)\right) d \hat{z}>\int_{t_{0}(z)}^{z} \sigma_{z}(z, \hat{z}) d \hat{t} \\
\sigma(z, t)>\sigma_{\text {slopel }}(z)
\end{gathered}
$$

FIG. 6. Schematic showing comparisons between actual stresses $\sigma(z, t)$ and $\sigma_{\text {slope }}(z)$, for films where the stress increases with increasing $z$ and $\sigma_{t}(z, t)$ $>0$. The integrals shown here appear in Eq. (7).

should produce tensile stress below the growth surface as deposition proceeds, such that $\sigma_{t}(z, t)>0$. A general explanation of how this will affect $\sigma(z, t)$ is depicted in Fig. 6, where two relative positions are shown. To understand this assessment, note that the two integrals in Eq. (7) have opposite signs. Thus their relative magnitudes determine whether $\sigma(z, t)$ exceeds $\sigma_{\text {slope }}(z)$ or vice versa. Near the substrate surface, the first integral in Eq. (7) spans a relatively small distance ( 0 to $z$ ), while the second integral spans a relatively large time $\left(t_{o}\right.$ to $\left.t\right)$. This relative difference implies that $\sigma(z, t)>\sigma_{\text {slope }}(z)$. The reverse will be true for larger $z$ values close to the top of the film, where the first integral encompasses a larger distance and the second integral spans a smaller time because $t_{o}(z)$ is larger. Both higher $\sigma(z, t)$ in the lower portion of the film and lower $\sigma(z, t)$ in the upper portion will decrease the total stress gradient across the film. Thus with $\sigma_{t}>0$, Eq. (7) indicates that the actual stress profile $\sigma(z, t)$ exhibits a smaller gradient than that inferred from only the $\sigma_{\text {slope }}(z)$ measurements. This is exactly what was observed in the experiments. Thus the hydrogen induced effects during plasma annealing are at least qualitatively consistent with the observed stress gradients. It should, however, be noted that the atomic hydrogen content of the growth environment is significantly lower than that in the pure hydrogen plasma that was used for annealing.

\section{Intrinsic stress evolution}

Phenomena that can induce intrinsic stress have been studied in a wide range of materials. In general, a number of factors can influence the intrinsic stress, including the deposition method, film microstructure, elastic properties, and surface and interface free energies. Thus, it is important to interpret experimental results with caution. In particular, 
$\mathrm{NCD}$ is a unique material where stress evolution has not been previously investigated in detail. To completely describe intrinsic stress evolution during deposition, both of the terms on the right hand side of Eq. (4) must be evaluated. This requires a more detailed analysis of $\sigma_{t}$, which is beyond the scope of the current paper. However, some consideration of $\sigma_{o}(z)$ with respect to existing theories of tensile growth stresses is still possible. To consider this quantity, Eq. (6) can be rearranged to obtain

$$
\sigma_{o}(z)=\sigma_{\text {slope }}(z)-\frac{1}{u} \int_{0}^{z} \sigma_{t}\left(\hat{z}, t_{o}(z)\right) d \hat{z} .
$$

Since both the plasma annealing and stress gradient results imply that $\sigma_{t}>0$, Eq. (8) indicates that $\sigma_{o}(z)$ $<\sigma_{\text {slope }}(z)$. Using the stress changes during plasma annealing as a rough guide, the magnitude of the last term in Eq. (8) is expected to be smaller than $\sigma_{\text {slope }}(z)$. Thus, the stresses produced directly by the growth process $\sigma_{o}(z)$ are relatively large and tensile. When films of comparable thicknesses are compared, higher stresses are still observed in those grown with higher $\mathrm{H}_{2}$ [e.g., see Fig. 4(b)]. Some of this stress increase is probably associated with the post-deposition processes discussed in section IV.B, and described by $\sigma_{t}$ in Eq. (8). It is also possible that the faster growth rates observed with more $\mathrm{H}_{2}$ lead to larger values of $\sigma_{o}$, since similar effects have been observed in other materials. ${ }^{44-46}$

In comparison with NCD, large tensile stresses in microcrystalline diamond have been attributed to the energy reduction that occurs when the free surfaces of two neighboring grains come together to form a grain boundary. ${ }^{15}$ This can cause neighboring grains to pull together, as proposed by Hoffman and co-workers. ${ }^{47,48}$ Several recent models of this phenomenon can be fit to the following form: ${ }^{49,50}$

$$
\frac{\langle\sigma\rangle}{E}=A\left(\frac{-\Delta \gamma}{E L}\right)^{B}
$$

where $L$ is the grain size and $\Delta \gamma=\gamma_{B}-2 \gamma_{S}$ is the energy reduction based on $\gamma_{S}$, the free energy of the island surface, and $\gamma_{B}$, the free energy of the grain boundary that forms between neighboring islands. The constants $A$ and $B$ depend on the island shape and contact geometry. During the growth process, the type of energy criteria that leads to Eq. (9) can also be incorporated into a finite element model (FEM) to obtain $\sigma_{o}(z) .^{51}$

Based on the data in Table I, NCD films with larger grain sizes exhibit higher stresses, however, Eq. (9) predicts the opposite trend. Also, values of $\sigma_{\text {slope }}[$ see Eq. (7) $]$ that are obtained from Fig. 4(b) and other similar experiments show higher stresses in films grown with higher $\mathrm{H}_{2}$, where grain sizes are larger [i.e., in contradiction to Eq. (9)]. Comparisons between microcrystalline diamond $(L \sim 1 \mu \mathrm{m})$ and NCD are also inconsistent with Eq. (9), because the observed stresses in microcrystalline films are comparable to or larger than the NCD values. ${ }^{15,19}$ Interpreting this difference solely with Eq. (9) requires that NCD exhibit $\Delta \gamma$ values that are substantially lower than those in the microcrystalline mate- rial (e.g., $\Delta \gamma=1 \mathrm{~J} / \mathrm{m}^{2}$ in microcrystalline diamond versus $\Delta \gamma<\sim 0.03 \mathrm{~J} / \mathrm{m}^{2}$ in NCD, with $B=\frac{1}{2}$ ). Values this low are highly unlikely.

Although Eq. (9) is not consistent with some of the observed trends in NCD, the surface/interface energy $(\Delta \gamma)$ term in Eq. (9) is still a likely driving force for the observed tensile values of $\sigma_{o}$. In addition to this initial tensile stress, hydrogen induced reactions appear to have a significant impact on the stresses in these NCD films, as outlined above. Other mechanisms may also produce additional tensile or compressive stresses.

\section{SUMMARY}

The CVD of nanocrystalline diamond films in $\mathrm{Ar} / \mathrm{H}_{2}$ plasmas produces significant tensile stresses. Substantial increases in these stresses occur during annealing in hydrogen plasmas. It is likely that hydrogen interactions with grain boundaries induce additional tensile stress, during plasma annealing and probably during the growth process as well. The results indicate that hydrogen apparently has a more important effect on residual stresses than does the grain size. Further experiments on these effects are needed. However, the observations made to date seem to contradict most existing models of tensile stress evolution, which predict a significant increase in stress as grain size decreases.

The ability to vary stress levels and stress gradients with hydrogen may provide important opportunities for engineering residual stresses in NCD films. This is particularly pertinent to MEMS devices where the films are not fully constrained, such that stress gradients can lead to significant bending. The ability to vary stress over hundreds of megapascals or more is also important in films where the proper control of residual stresses is necessary to prevent mechanical failures. Because the hydrogen induced stress changes appear to be associated with grain boundaries, the ability to exercise substantial control of residual stresses is a direct result of the nanocrystalline structure of these materials.

\section{ACKNOWLEDGMENTS}

We thank Ryo Saotome and Douglas Romanoff for their assistance in conducting the experiments. Primary research support from the National Science Foundation, under Award No. DMR-0305418 is gratefully acknowledged. This work also made use of MRSEC Shared Experimental Facilities, supported by NSF under Award No. DMR-0079964.

${ }^{1}$ D. M. Gruen, Annu. Rev. Mater. Sci. 29, 211 (1999).

${ }^{2}$ C. Zuiker et al., Thin Solid Films 270, 154 (1995).

${ }^{3}$ A. Erdemir, G. R. Fenske, A. R. Krauss, D. M. Gruen, T. Mc Cauley, and R. T. Csencsits, Surf. Coat. Technol. 565, 120 (1999).

${ }^{4}$ P. Hollman, O. Wänstrand, and S. Hogmark, Diamond Relat. Mater. 7, 1471 (1998).

${ }^{5}$ R. L. C. Wu, A. K. Rai, A. Garscadden, P. Kee, H. D. Desai, and K. Miyoshi, J. Appl. Phys. 72, 110 (1992).

${ }^{6}$ A. R. Krauss et al., J. Appl. Phys. 89, 2958 (2001).

${ }^{7}$ W. Yang et al., Nat. Mater. 1, 253 (2002).

${ }^{8}$ H. D. Espinosa et al., Exp. Mech. 43, 256 (2003).

${ }^{9}$ H. D. Espinosa, B. Peng, B. C. Prorok, N. Moldovan, O. Auciello, J. A. Carlisle, D. M. Gruen, and D. C. Mancini, J. Appl. Phys. 94, 6076 (2003).

${ }^{10}$ H. D. Espinosa and B. Peng, Proceedings of the 4th International Symposium on MEMS and Nanotechnology (Society of Experimental Mechan- 
ics, Bethel, CT, 2003, p.307.

${ }^{11}$ T. A. Friedmann, J. P. Sullivan, J. A. Knapp, D. R. Tallant, D. M. Follstaedt, D. L. Medlin, and P. B. Mirkarimi, Appl. Phys. Lett. 71, 3820 (1997).

${ }^{12}$ M. Chhowalla, Y. Yin, G. A. J. Amaratunga, D. R. McKenzie, and T. Fraurnheim, Appl. Phys. Lett. 69, 2344 (1996).

${ }^{13}$ R. G. Lacerda and F. C. Marques, Appl. Phys. Lett. 73, 617 (1998).

${ }^{14} \mathrm{H}$. Windischmann and K. J. Gray, Diamond Relat. Mater. 4, 837 (1995).

${ }^{15}$ B. W. Sheldon, K. K. A. Lau, and A. Rajamani, J. Appl. Phys. 90, 5097 (2001).

${ }^{16}$ S. Nijhawan, S. M. Jankovsky, B. W. Sheldon, and B. L. Walden, J. Mater. Res. 14, 1046 (1999).

${ }^{17}$ S. Nijhawan, Ph.D. thesis, Brown University, 1999.

${ }^{18}$ L. Bergman and R. J. Nemanich, J. Appl. Phys. 78, 6709 (1995).

${ }^{19}$ A. Rajamani, B. W. Sheldon, S. Nijhawan, A. Schwartzman, J. Rankin, B. L. Walden, and L. Riester, J. Appl. Phys. 96, 3531 (2004).

${ }^{20}$ E. Chason and B. W. Sheldon, Surf. Eng. 19, 387 (2003).

${ }^{21}$ G. G. Stoney, Proc. R. Soc. London, Ser. A 82, 172 (1909).

${ }^{22}$ A. Rajamani, Ph D. thesis, Brown University, 2003.

${ }^{23}$ T. G. Bifano, H. T. Johnson, P. Bierden, and R. K. Mali, J. Microelectromech. Syst. 11, 592 (2002).

${ }^{24}$ D. Zhou, D. M. Gruen, L. C. Qin, T. G. McCauley, and A. R. Krauss, J. Appl. Phys. 84, 1981 (1998).

${ }^{25}$ A. N. Jones, W. Ahmed, I. U. Hassan, C. A. Rego, H. Sein, M. Amar, and M. J. Jackson, J. Phys.: Condens. Matter 15, S2969 (2003).

${ }^{26}$ T. Lin, G. Y. Yu, A. T. S. Wee, Z. X. Shen, and K. P. Loh, Appl. Phys. Lett. 77, 2692 (2000).

${ }^{27}$ L. C. Qin, D. Zhou, A. R. Krauss, and D. M. Gruen, Nanostruct. Mater. 10, 4 (1998).

${ }^{28}$ P. Keblinski, D. Wolf, S. R. Phillpot, and H. Gleiter, J. Mater. Res. 13, 2077 (1998).

${ }^{29}$ N. Wada, P. J. Gaczi, and A. Solin, J. Non-Cryst. Solids 35\&36, 543 (1980).

${ }^{30}$ S. R. Salis, D. J. Gardiner, M. Bowden, J. Savage, and D. Rodway, Dia- mond Relat. Mater. 5, 589 (1996).

${ }^{31}$ J. Birrell, J. E. Gerbi, O. Auciello, J. M. Gibson, J. Johnson, and J. A. Carlisle, Diamond Relat. Mater. 14, 86 (2005).

${ }^{32}$ R. Pfeiffer, H. Kuzmany, N. Salk, and B. Gunther, Appl. Phys. Lett. 82, 4149 (2003).

${ }^{33}$ A. C. Ferrari and J. Robertson, Phys. Rev. B 63, 121405(R) (2001).

${ }^{34}$ H. Kuzmany, R. Pfeiffer, N. Salk, and B. Günther, Carbon 42, 911 (2004).

${ }^{35}$ A. C. Ferrari and J. Robertson, Philos. Trans. R. Soc. London, Ser. A 362, 2477 (2004).

${ }^{36}$ A. C. Ferrari and J. Robertson, Phys. Rev. B 64, 075414 (2001).

${ }^{37}$ F. Tuinstra and J. L. Koening, J. Chem. Phys. 53, 1126 (1970).

${ }^{38}$ A. C. Ferrari and J. Robertson, Phys. Rev. B 61, 14095 (2000).

${ }^{39}$ A. Rajamani, R. Beresford, and B. W. Sheldon, Appl. Phys. Lett. 79, 3776 (2001).

${ }^{40}$ C. L. Chen, C. T. Chia, C. C. Chiu, and I. N. Lin, Diamond Relat. Mater. 11, 262 (2002).

${ }^{41}$ Y. Hayashi, D. Mori, T. Soga, and T. Jimbo, Phys. Solid State 46, 714 (2004).

${ }^{42}$ L. C. Qin, D. Zhou, A. R. Krauss, and D. M. Gruen, Nanostruct. Mater. 10, 649 (1998).

${ }^{43}$ V. D. Frolov, A. V. Karabutov, V. I. Konov, S. M. Pimenov, and A. M. Prokhorov, J. Phys. D 32, 815 (1999).

${ }^{44}$ B. W. Sheldon, A. Rajamani, A. Bhandari, E. Chason, S. K. Hong, and R. Beresford, J. Appl. Phys. 98, 043509 (2005).

${ }^{45}$ E. Chason, B. W. Sheldon, L. B. Freund, J. A. Floro, and S. J. Hearne, Phys. Rev. Lett. 88, 156103 (2002).

${ }^{46}$ S. J. Hearne and J. A. Floro, J. Appl. Phys. 97, 014901 (2005).

${ }^{47}$ J. D. Finegan and R. W. Hoffman, J. Appl. Phys. 30, 587 (1959).

${ }^{48}$ F. A. Doljack and R. W. Hoffman, Thin Solid Films 12, 71 (1972).

${ }^{49}$ W. D. Nix and B. M. Clemens, J. Mater. Res. 14, 3467 (1999).

${ }^{50}$ L. B. Freund and E. Chason, J. Appl. Phys. 89, 4866 (2001).

${ }^{51}$ A. Rajamani, B. W. Sheldon, E. Chason, and A. F. Bower, Appl. Phys. Lett. 81, 1204 (2002). 